\title{
Modelling of hydrogen diffusion in the retina
}

\author{
W. F. Mansoor ${ }^{1} \quad$ G. C. Hocking ${ }^{2} \quad$ D. E. Farrow ${ }^{3}$
}

(Received 8 January 2020; revised 14 May 2020)

\begin{abstract}
A simple mathematical model for diffusion of hydrogen within the retina has been developed. The model consists of three, well-mixed, one dimensional layers that exchange hydrogen via a diffusion process. A Fourier series method is applied to compute the hydrogen concentration. The effect of important parameters is examined and discussed. The results may contribute to an understanding of the hydrogen clearance technique to estimate blood flow. A two dimensional numerical method for the hydrogen diffusion is also presented. It is shown that the predominant features of the process are captured quite well by the simpler model.
\end{abstract}

\section{Contents}

\section{Introduction}

DoI:10.21914/anziamj.v61i0.14995, C Austral. Mathematical Soc. 2020. Published 2020-07-07, as part of the Proceedings of the 14th Biennial Engineering Mathematics and Applications Conference. ISSN 1445-8810. (Print two pages per sheet of paper.) Copies of this article must not be made otherwise available on the internet; instead link directly to the DOI for this article. 
3 Results

C122

4 Two dimensional model

C129

5 Final remarks

C133

References

C134

\section{Introduction}

The process of vision relies on the retina, where light waves are turned into neurochemical and electrical signals. Blood flow in the retina is an important signal in the diagnosis of a number of dangerous diseases that may cause loss of sight including glaucoma and diabetic retinopathy $[2,4,5]$. The retina consists of numerous anatomical layers. On the internal surface of the retina is the retinal circulation, while on the exterior surface of the retina is the choroidal vasculature [10]. The avascular layer is located between these two layers and relies on diffusion of metabolites through tissue. The choroid vasculature requires a larger amount of blood flow than the retinal vasculature and consumes a higher amount of oxygen. The inclusion of the vascular retina extends the work of Farrow et al. [3], and comparing the two models reflects the difference between the eyes of guinea pigs, who have no retinal flow, with rats and humans who do $[12,13]$.

One experimental procedure to estimate blood flow is the hydrogen clearance technique, in which a bolus of hydrogen saturated saline solution is introduced to the blood supply and then measured across the retina [1, 11]. This method was found to give highly reproducible concentration traces. Hydrogen is a conservative tracer, and the subsequent decay of the hydrogen concentration may be used to estimate the blood flow using the so-called 'hydrogen clearance curves' [7]. 
Figure 1: Schematic of the model for hydrogen exchange through the retina.

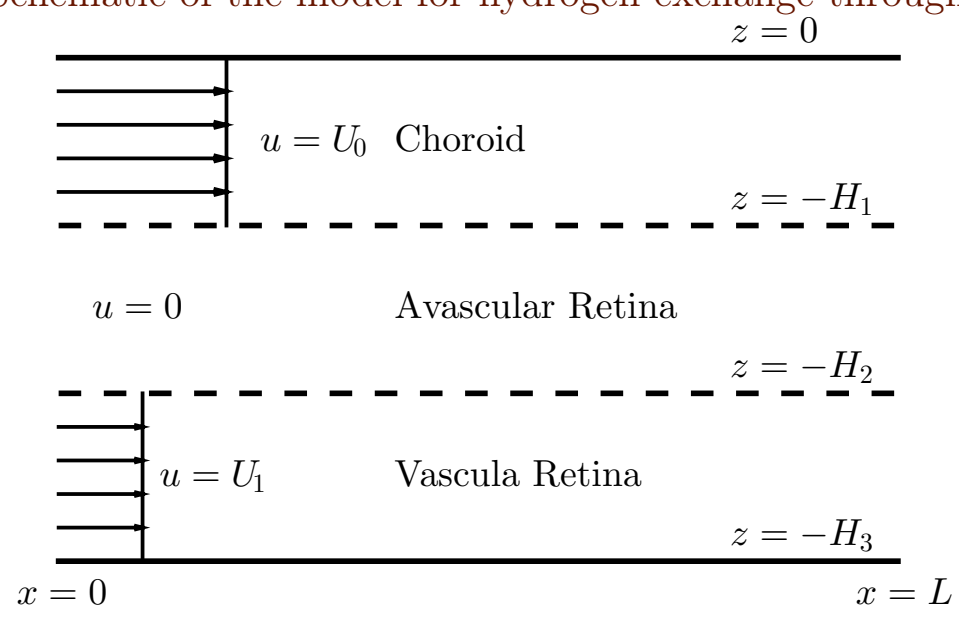

\section{Problem formulation and solution}

In this model the choroid, the avascular retina and the vascular retina are considered as well-mixed layers. The blood in the choroid and vascular retina are assumed to be moving at constant and uniform speeds $\mathrm{U}_{0}$ and $\mathrm{U}_{1}$, respectively, and there is no flow in the avascular layer. In both flowing layers there is a mesh of blood vessels and so an assumption of uniform flow is reasonable. In general, one expects $\mathrm{U}_{0}>\mathrm{U}_{1}$ as the retinal vasculature is sparser. This model is the same as the two-layer model of Farrow et al. [3] if the third layer velocity is removed $\left(U_{1}=0\right)$. We define $C(x, t), R(x, t)$ and $B(x, t)$ to be the concentration of hydrogen in the choroid, avascular retina and vascular retina, respectively. Figure 1 shows the main features of the model.

In non-dimensional form, the model equations are

$$
\begin{aligned}
& \frac{\partial C}{\partial t}+\frac{\partial C}{\partial x}=-\alpha(C-R), \\
& \frac{\partial R(x, t)}{\partial t}=\alpha(C-R)+\alpha(B-R),
\end{aligned}
$$




$$
\frac{\partial B}{\partial t}+\gamma \frac{\partial B}{\partial x}=-\alpha(C-R),
$$

where the velocity ratio is $\gamma=\mathrm{U}_{1} / \mathrm{U}_{0}$, the non-dimensional exchange parameter is $\alpha=\lambda l / \mathrm{U}_{0}, \lambda$ is the diffusivity, and $l$ is a length scale related to the initial size of the bolus of hydrogen. In this model the 'diffusion' between layers is handled by the exchange terms on the right sides of equations (1), (2) and (3) with strength given by 'diffusion' $\alpha$. The initial conditions are

$$
\mathrm{C}(\mathrm{x}, 0)=\frac{1}{\pi} e^{-x^{2}}, \quad \mathrm{~B}(x, 0)=0 \text { and } \mathrm{R}(x, 0)=0,
$$

on $0<x<\mathrm{L}$, where $\mathrm{C}(\mathrm{x}, 0)$ is the initial concentration of hydrogen in the choroid and the other two layers initially have no hydrogen. We seek a solution using complex Fourier series, that is,

$$
\begin{aligned}
& \mathrm{C}(x, \mathrm{t})=\sum_{k=-\infty}^{\infty} \mathrm{C}_{k}(\mathrm{t}) e^{i \beta_{k} x}, \quad \mathrm{R}(x, \mathrm{t})=\sum_{k=-\infty}^{\infty} \mathrm{R}_{k}(\mathrm{t}) e^{i \beta_{k} x}, \\
& \mathrm{~B}(x, \mathrm{t})=\sum_{k=-\infty}^{\infty} \mathrm{B}_{k}(\mathrm{t}) e^{i \beta_{k} x},
\end{aligned}
$$

where $\beta_{k}=2 \pi k / L$. The periodicity of the Fourier terms (5) has no impact on the results, provided successive intervals do not interact; that is, $\mathrm{U}_{0} \mathrm{~T} \ll \mathrm{L}$. Substituting (5) into (1), (2) and (3) with the conditions (4) yields a system of linear ODEs for the coefficients, written in matrix form as

$$
\frac{d \hat{C}_{k}}{d t}=A_{k} \hat{C}_{k}, \quad k=1,2, \ldots, N
$$

where

$$
A_{k}=\left[\begin{array}{ccc}
-\left(\alpha+\frac{2 \pi i k}{L}\right) & \alpha & 0 \\
\alpha & \alpha & 0 \\
0 & \alpha & -\left(\alpha+\frac{2 \pi i k}{L}\right)
\end{array}\right] \quad \text { and } \quad \hat{C}_{k}=\left[\begin{array}{c}
C_{k} \\
R_{k} \\
B_{k}
\end{array}\right]
$$

This system was solved using a linear multi-step method (routine lsode in Octave [6]). 
Figure 2: Profiles of $C(x, t), R(x, t)$ and $B(x, t)$ with $\alpha=0.2$ at different times for velocity ratio $\gamma=0.5$. Profiles are shown from top to bottom: the choroid, the avascular retina and the vascular retina (the profiles are vertically offset to make them clearer and in all cases the base level is zero concentration).

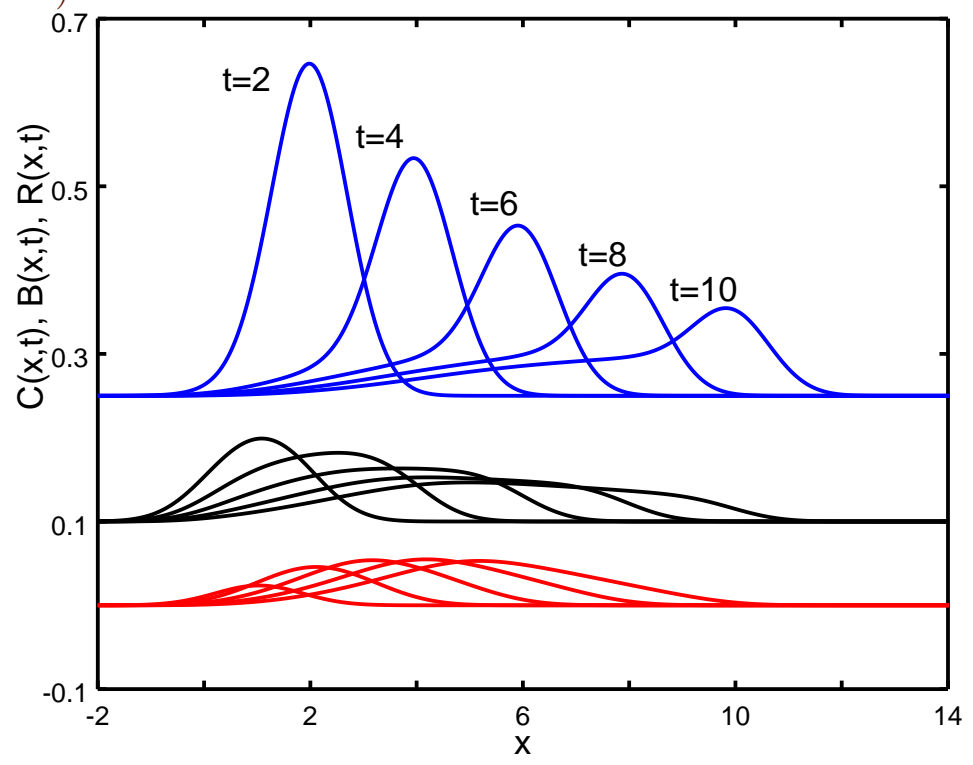

\section{Results}

Figure 2 shows a series of profiles of $C, R$ and $B$, when the velocity ratio is $\gamma=0.5$ with a low value of diffusion $\alpha=0.2$. The original bolus of hydrogen travels along in the choroid at the speed of flow, slowly diffusing into the avascular retina and then the vascular retina. The tail fattens as the lagging concentration in the avascular retina begins to return to the choroid. This effect is also seen in the avascular region as there is a return flow from the vascular retina. The peak in the choroid dwindles quickly as it travels along, while the peak in the vascular retina increases.

Figure 3 shows profiles for $\gamma=0.5$, but with a higher value of diffusion, 
Figure 3: Profiles of $C(x, t), R(x, t)$ and $B(x, t)$, with $\alpha=0.3$ at different times with $\gamma=0.5$. Profiles are shown from top to bottom, the choroid, the avascular retina and the vascular retina (offset as in Figure 2).

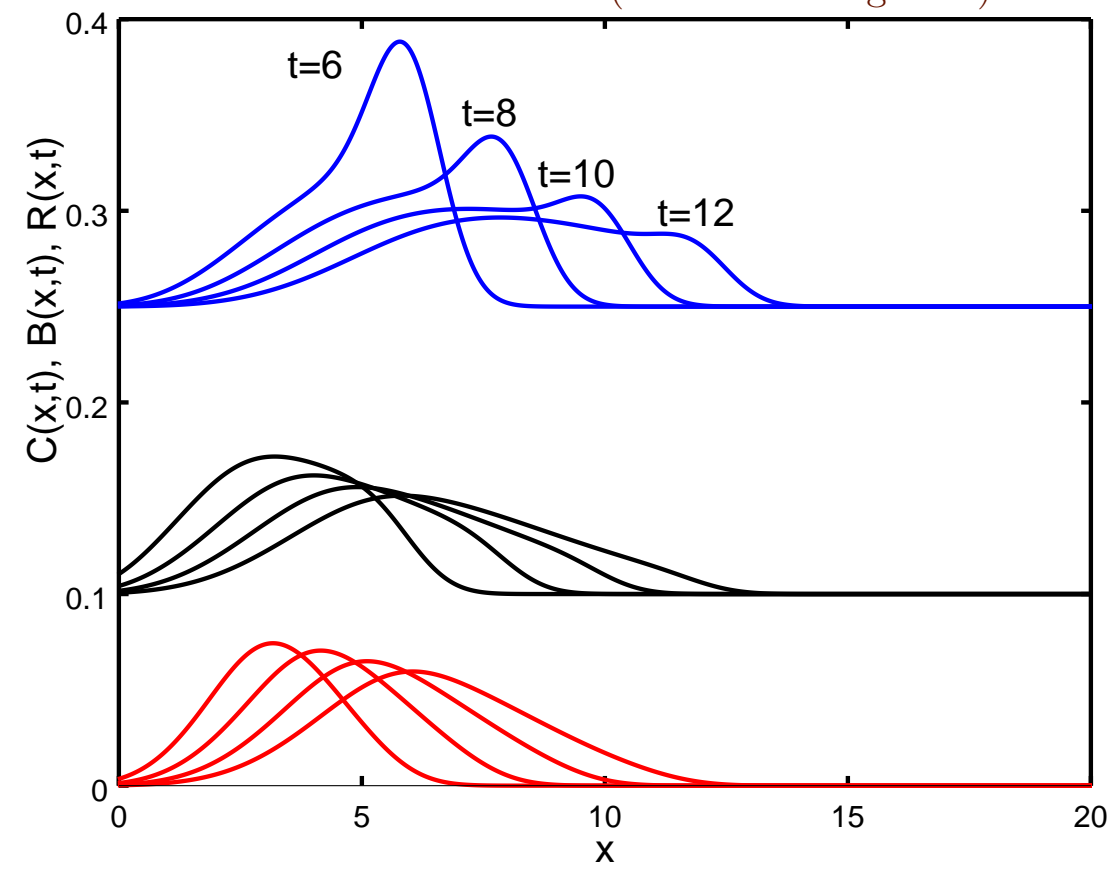

$\alpha=0.3$. Here, the return flow from the avascular retina causes a more dramatic fattening of the tail in the choroid so that by $t=12$ the highest concentration value has moved back from the location of the original bolus to a point in the tail. The original peak is still very clear at $t=6$ and $t=8$ but at $t=10$ the tail maximum is similar in height to the original peak and by $t=12$ it has exceeded the original peak.

Figure 4 shows the evolution of the location $x_{m}$ of the highest value of hydrogen in each of the layers at different velocity ratios, $\gamma=0$ (top) and $\gamma=1$ (bottom), for $\alpha=0.3$. The position of the maximum value initially moves at the speed of flow, but as reverse diffusion occurs the maximum 
Figure 4: The location of the peak concentration $x_{m}$ in the choroid (dashed blue line), the avascular retina (the solid green) and the vascular retina (the dash-dot red) for $\alpha=0.3$. The top panel is $\gamma=0$ and the bottom is $\gamma=1$.
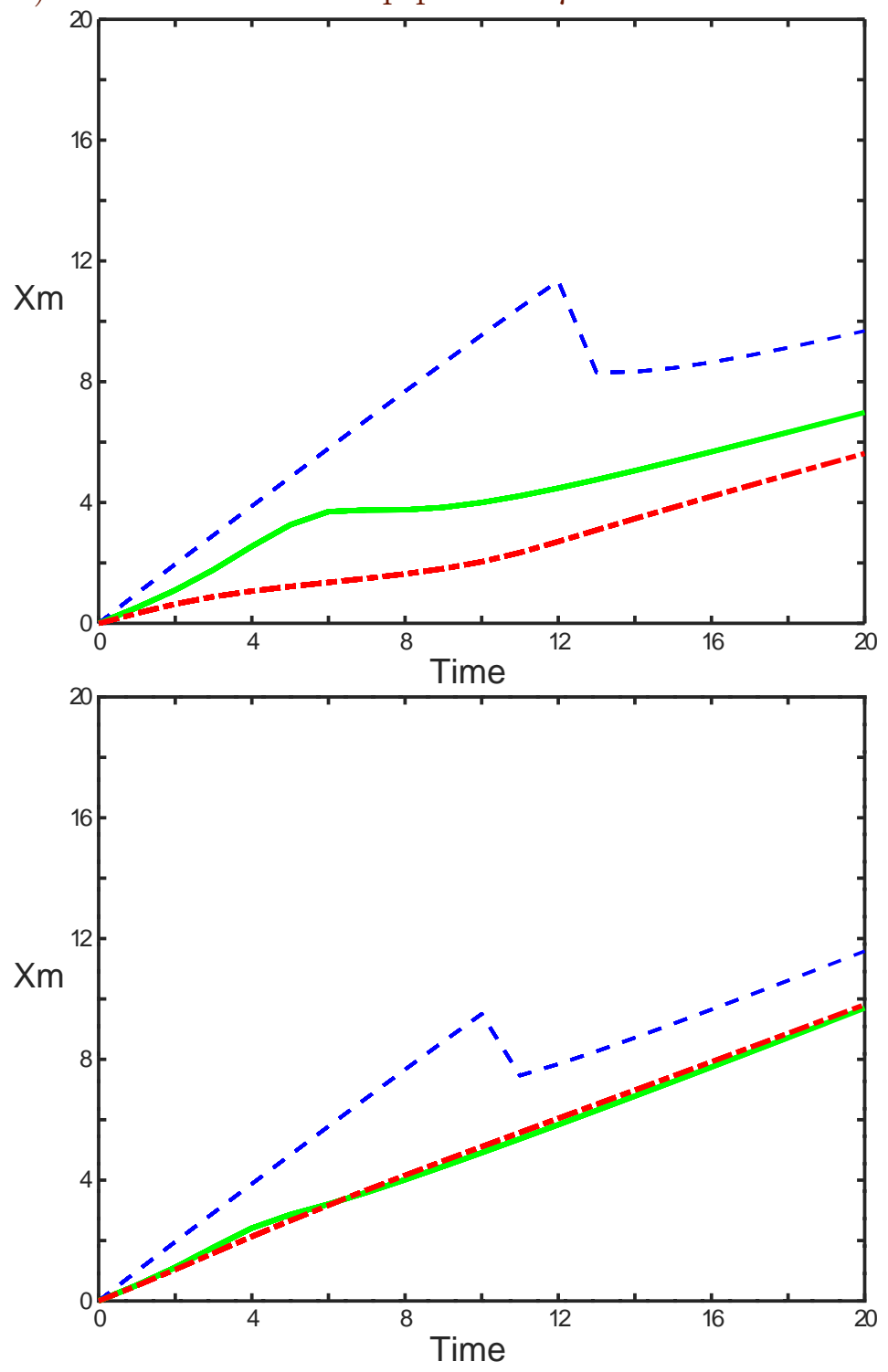
jumps backward to a position in the tail, and then moves forward at a slower speed. The sharp jump back in the choroid is matched by a smoother, but earlier, transition in the other two layers. The presence of the vascular retina, where $\gamma \neq 0$, leads to an earlier transition time.

Figure 5 shows the location $x_{m}$ of the peak value in the three layers for a large diffusion $\alpha=0.5$, for $\gamma=0$ (top) and $\gamma=1$ (bottom). This shows the impact of a strong interchange between the layers. The results show the same qualitative behaviour as the results for a lower value of $\alpha$, but the jump in the position of the peak concentration occurs much earlier due to the more rapid rate of hydrogen flux into the vascular retina. In the choroid with $\gamma=0$ we see the shift from the primary, original peak to the second peak situated further back in the profile at around $t=7$, and the same effect at around $t=4$ in the avascular retina. When $\gamma=1$ the shift occurs at about $t=6 \mathrm{in}$ the choroid, but is almost immediate in the other two layers.

Figure 6 shows the velocity of the point of peak hydrogen concentration in the choroid for different values of diffusion $\alpha$ and vascular flow velocity $\gamma=0$ and $\gamma=1$. This reinforces the points discussed above, but also shows that the final advection speed for all $\alpha$ is the same for a given $\gamma$ value. For lower values of $\alpha$ the peak shift happens at a later time with a sharp jump, while for larger values of $\alpha$ the shift occurs at an earlier time and smoothly. Extending the analysis of Mitchell [9] to three layers, we add the three equations (1), (2) and (3) giving

$$
\mathrm{C}_{\mathrm{t}}+\mathrm{R}_{\mathrm{t}}+\mathrm{B}_{\mathrm{t}}+\mathrm{C}_{\mathrm{x}}+\gamma \mathrm{B}_{\mathrm{x}}=0 .
$$

After some time it is reasonable to assume that $\bar{C}=C=R=B$, giving

$$
\overline{\mathrm{C}}_{\mathrm{t}}+\frac{(1+\gamma)}{3} \overline{\mathrm{C}}_{\mathrm{x}}=0 \text {. }
$$

This provides a formula for the final advection velocity

$$
\mathrm{V}_{\mathrm{term}}=\frac{(1+\gamma)}{3}
$$

These final advection velocity values agree very well with those obtained in this work. Note that the final velocity is independent of the diffusion $\alpha$, as 
Figure 5: The location of the peak concentration $x_{m}$ in the choroid (dashed blue line), the avascular retina (the solid green) and the vascular retina (the dash-dot red) for $\alpha=0.5$. The top panel is $\gamma=0$ and the bottom is $\gamma=1$.
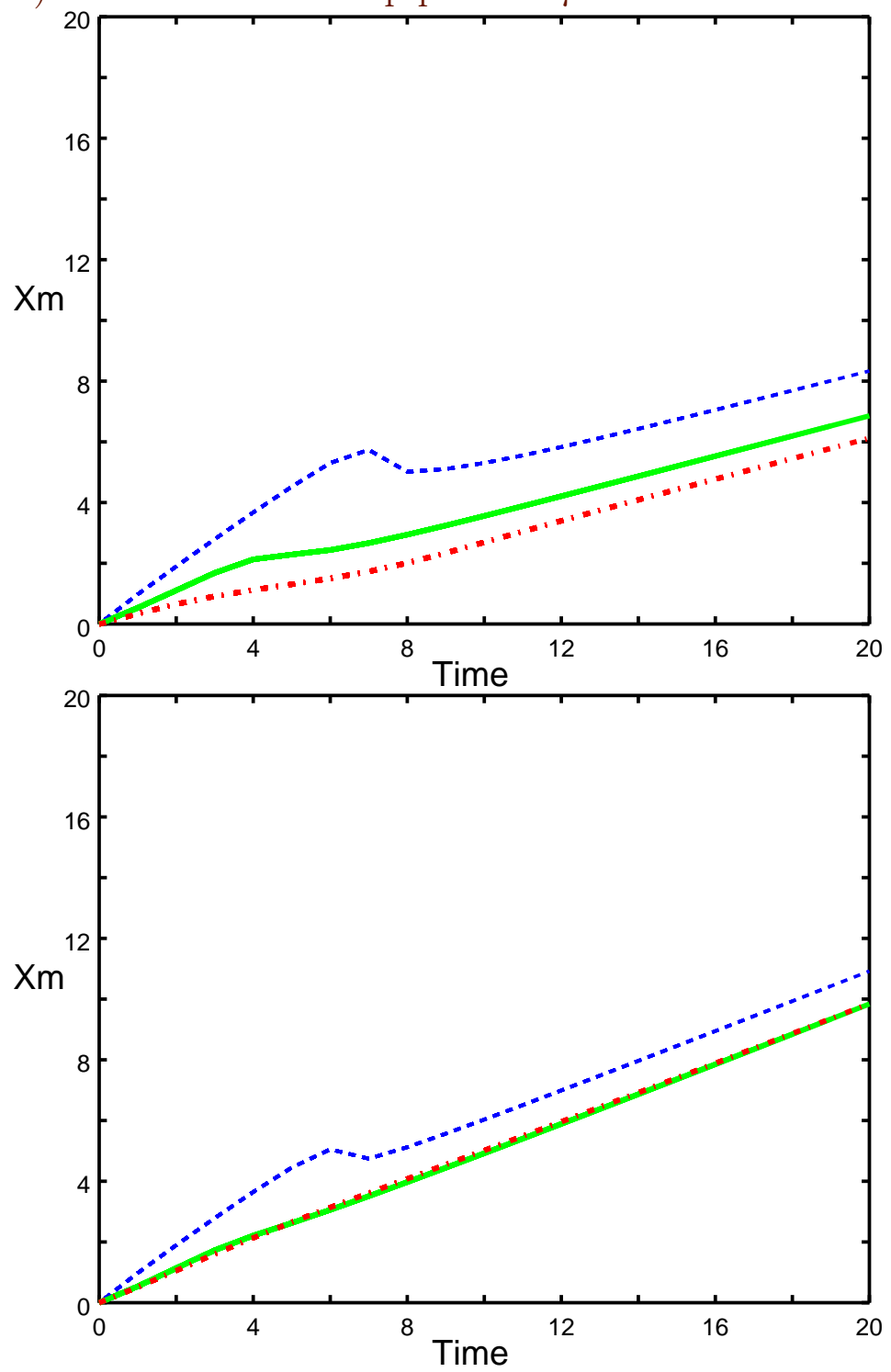
Figure 6: The advection velocity of the peak in the choroid for different $\alpha$. In both cases $\alpha$ has six values which are $\alpha=0.2,0.3,0.4,0.5,1$ and respectively at various values of $\gamma=0$ (top) and $\gamma=1$ (bottom).
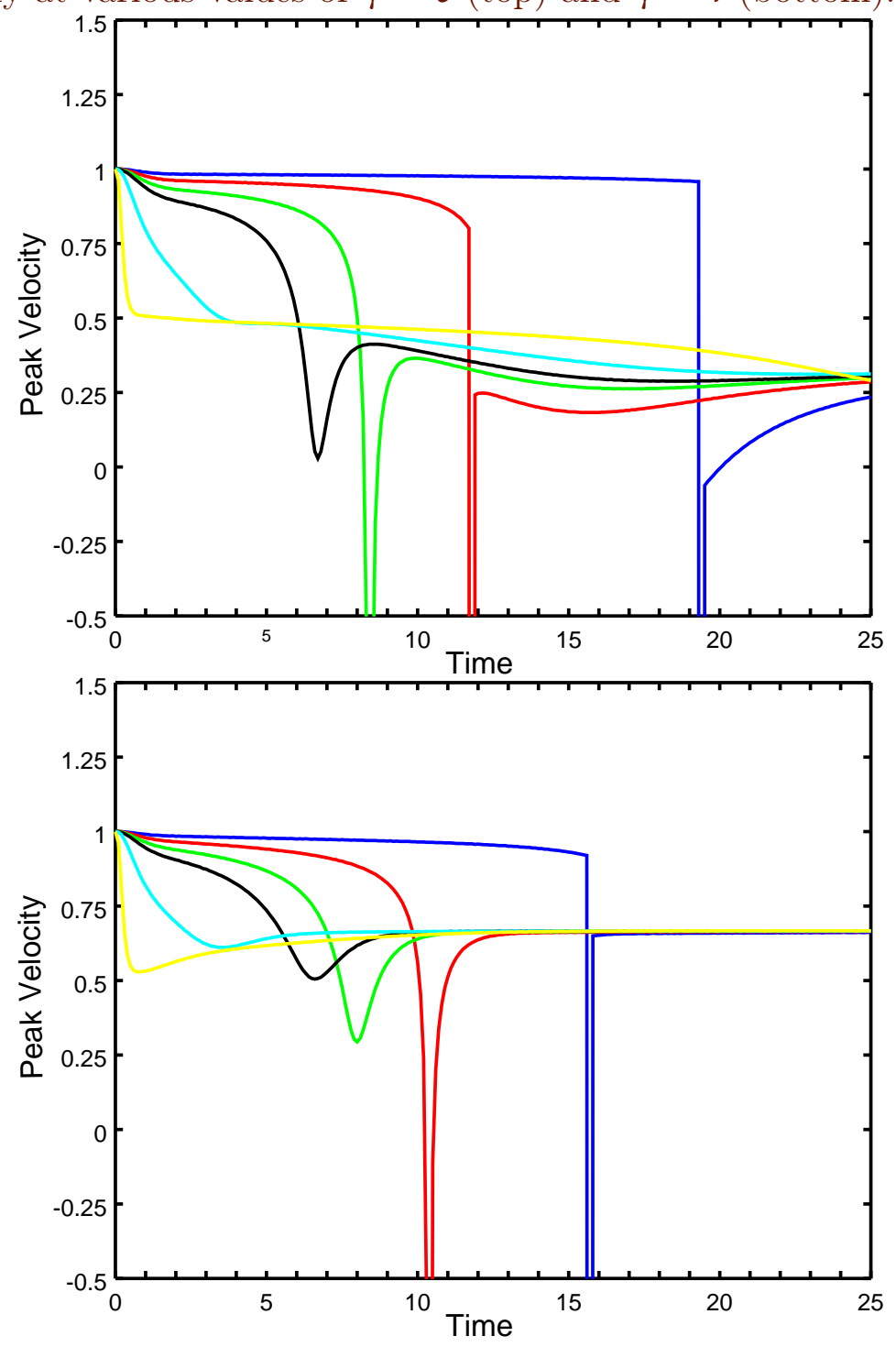
$\alpha$ effects the timing and severity of the transition event, but not the final velocity.

\section{Two dimensional model}

In the 2D model we treat the layers as a single region with different speeds in the three layers. The model equation is

$$
\frac{\partial C}{\partial t}+\mathrm{U}(z) \frac{\partial \mathrm{C}}{\partial x}=\frac{1}{\mathrm{Pe}}\left(\frac{\partial^{2} \mathrm{C}}{\partial x^{2}}+\frac{\partial^{2} \mathrm{C}}{\partial z^{2}}\right)
$$

where $\mathrm{C}(x, z, t)$ is the hydrogen concentration, $\mathrm{Pe}=\mathrm{Ul} / \mathrm{k}$ is the Peclet number, $\mathrm{k}$ is the coefficient of diffusion in the eye tissue and the non-dimensional velocity is

$$
\mathrm{U}(z)= \begin{cases}1, & \text { for }-\mathrm{H}_{1}<z \leqslant 0, \\ 0, & \text { for }-\mathrm{H}_{2}<z \leqslant-\mathrm{H}_{1}, \\ \gamma, & \text { for }-\mathrm{H}_{3} \leqslant z \leqslant-\mathrm{H}_{2},\end{cases}
$$

where $\mathrm{H}_{1}<\mathrm{H}_{2}<\mathrm{H}_{3}$. The choroid occupies the layer $-\mathrm{H}_{1}<z<0$, the avascular retina occupies $-\mathrm{H}_{2}<z<-\mathrm{H}_{1}$ and the vascular retina occupies $-\mathrm{H}_{3}<z<-\mathrm{H}_{2}$ (Figure 1). The three layers are assumed to be of equal thickness, so that $\mathrm{H}_{1}=1, \mathrm{H}_{2}=2, \mathrm{H}_{3}=3$. The boundary conditions are that the concentration is periodic in the $x$-direction and there is no vertical flux at $z=0$ and $z=-\mathrm{H}_{3}$. The initial condition consists of a sine profile bolus of hydrogen in the choroid, that is

$$
C(x, z, 0)= \begin{cases}-\sin (2 \pi x) \sin \left(2 \pi z / H_{1}\right), & \text { for } 0 \leqslant x \leqslant 1,-H_{1} \leqslant z \leqslant 0 \\ 0, & \text { otherwise. }\end{cases}
$$

The two dimensional model is resolved numerically using a finite volume method. Quadratic upwinding is used for the advection term, following Leonard [8], and a standard centred difference is utilized for the diffusion term. A large system of ODEs in time was obtained that was solved with the Octave routine lsode. 
Figure 7: Hydrogen concentration for $t=0,10$ and 20 and $\mathrm{Pe}=11, \gamma=0$.
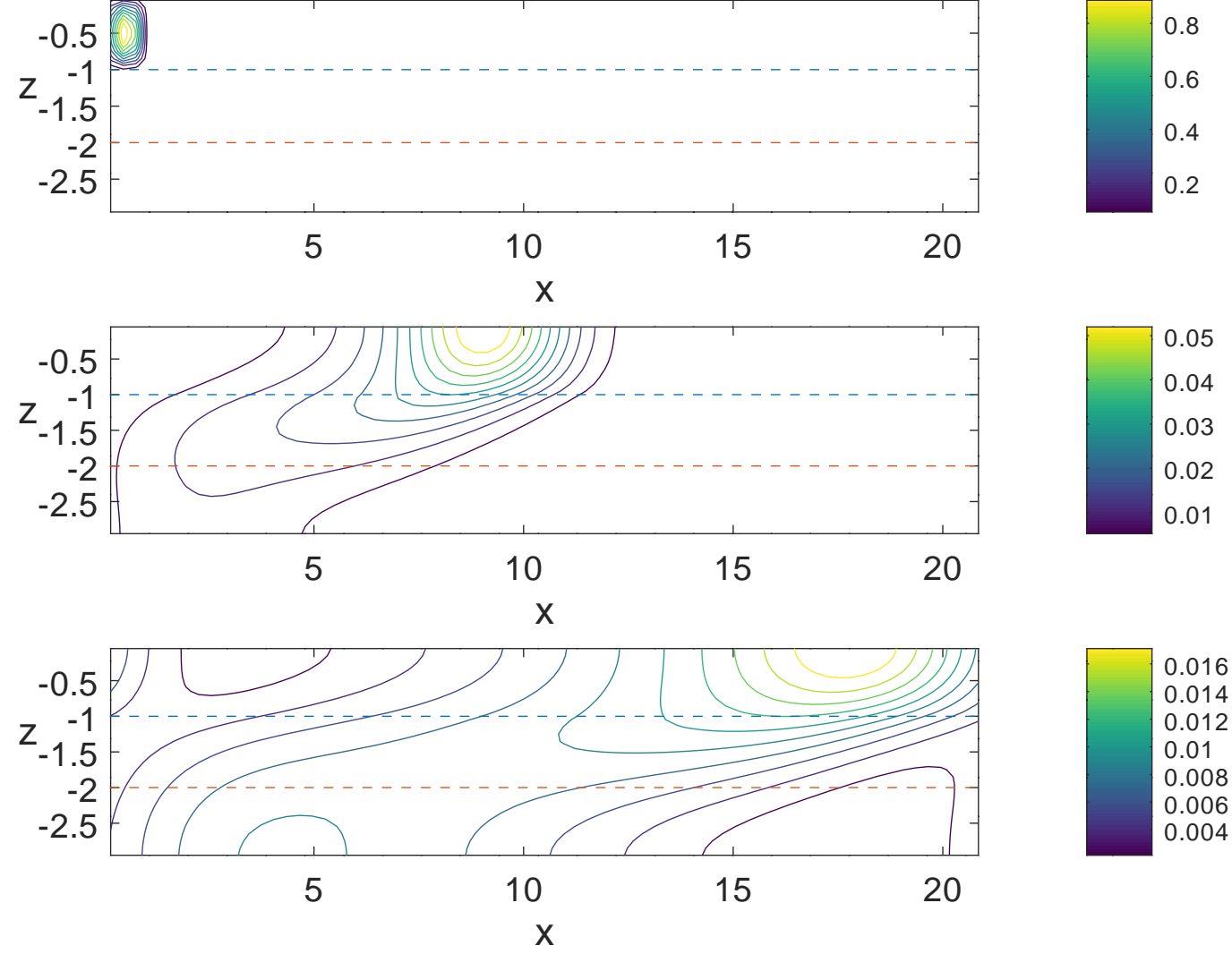

Figure 7 shows contours of hydrogen for large $P e=11$ at $t=0,10$ and $t=20$ for $\gamma=0$. Since the avascular retina has no flow and the diffusion is small, the retinal layers lag behind the choroid at $t=10$. When $t=20$ the peak concentration is still lagging, but we find that there is a local maximum in the choroid at $x \approx 17$, and another in the vascular retina at $x \approx 4$.

Figure 8 gives comparable contours to those of Figure 7 but with $\gamma=0.5$. The lag effect is still seen, but it is not as significant. After about $t \approx 10$ the propagation rate within all three layers is approximately the same. At $t=20$ 
Figure 8: Hydrogen concentration for $t=0,10$ and 20 and $\mathrm{Pe}=11, \gamma=0.5$.
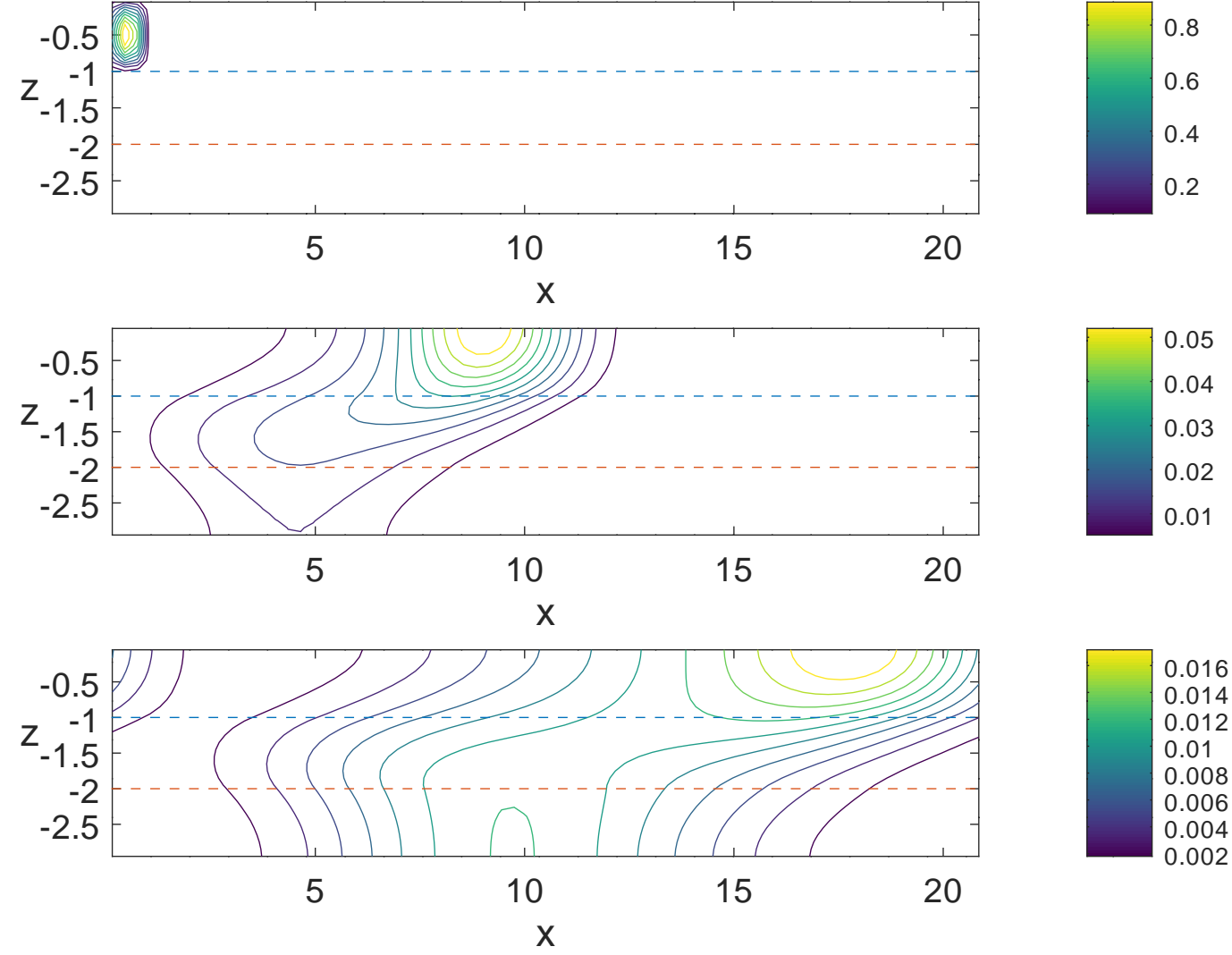

there are still two maxima, one in the choroid at $x \approx 17$ and the other in the vascular retina at $x \approx 10$. The reason for this is that, as the boundary conditions prevent flow out, the hydrogen builds to a maximum in the vascular retina and then flows back to the choroid leaving a high point in the vascular retina. Meanwhile, we see the bolus travel along with a fattening tail, as in the 1D model. The center of mass of hydrogen remains in the choroid as the diffusion is quite small. Thus advection dominates and diffusion is small, so a large Peclet number is comparable a small value of $\alpha$ in the $1 \mathrm{D}$ model. 
Figure 9: Hydrogen concentration for $t=0,10$ and 20 and $\mathrm{Pe}=1, \gamma=0$.
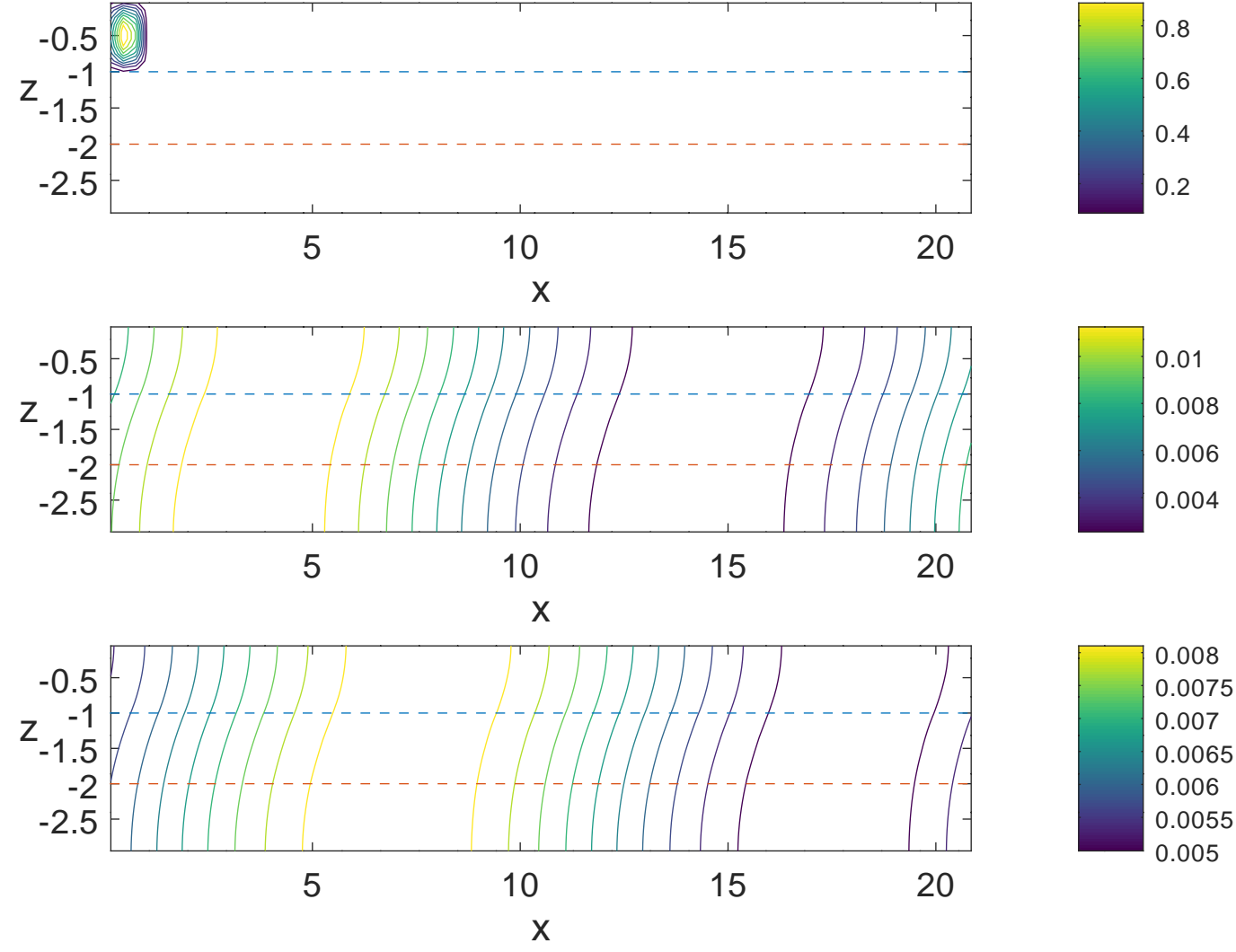

Figures 9 and 10 show comparable contours to those of Figures 7 and 8 but for small $P e=1$. This small Peclet number is equivalent to a large $\alpha$ in the 1D model. Here, the diffusion term plays a vital role in the transport of the hydrogen. The bolus of hydrogen moves through the choroid but quickly diffuses to the other layers after which it diffuses back to the choroid. By $t=10$ the distribution of hydrogen in the three layers has a clear symmetry with approximately equal amounts of hydrogen in each layer, and this appears to be maintained at least up to $t=20$. The arrival time of the hydrogen peak concentration to a particular location is significantly delayed compared 
Figure 10: Hydrogen concentration for $t=0,10$ and 20 and $\mathrm{Pe}=1, \gamma=0.5$.
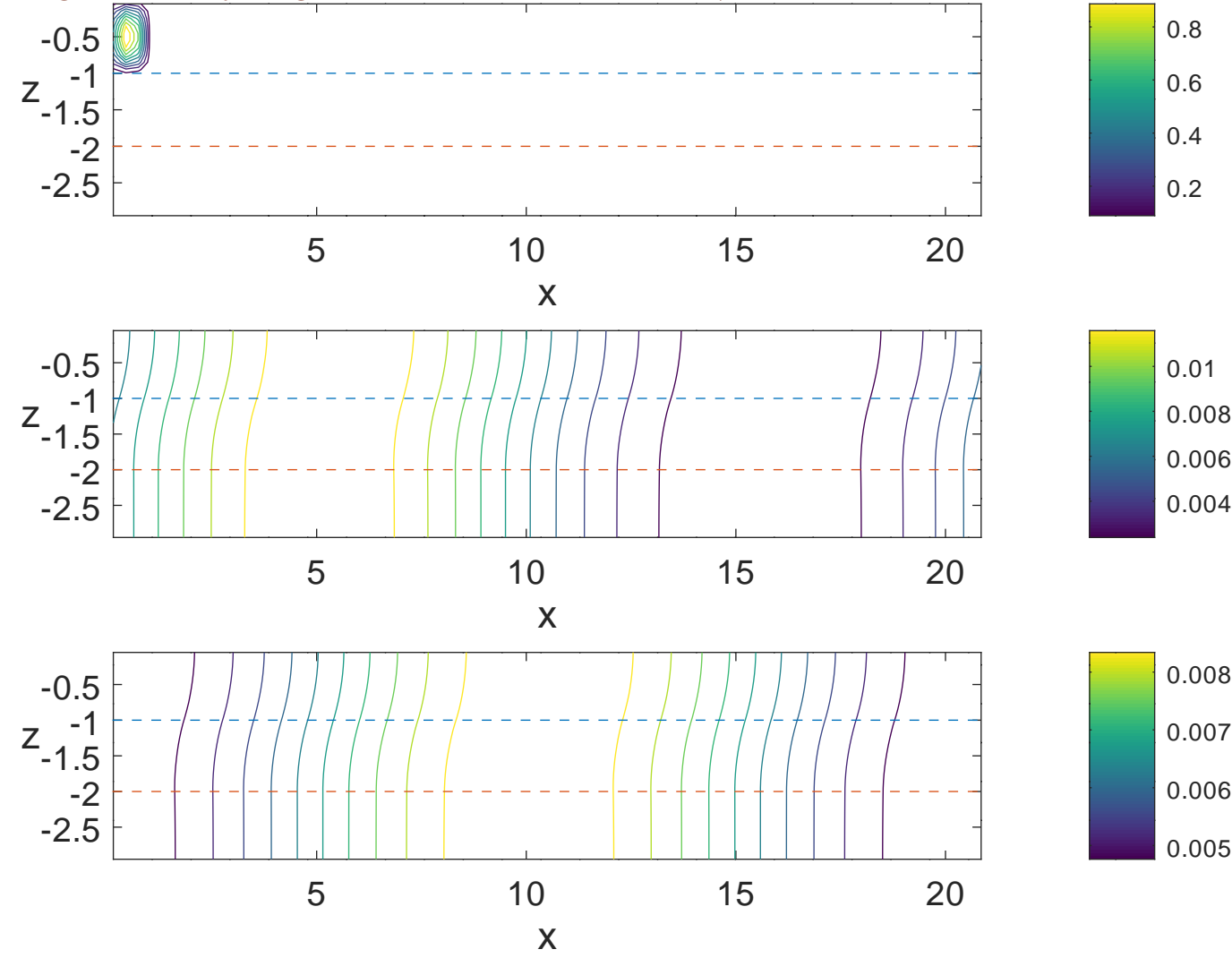

to the blood velocity, and therefore the speed of travel of the hydrogen peak in this case is approximately 0.2 for $\gamma=0$, and 0.5 for $\gamma=0.5$. This is the same effect as the slowly traveling concentration peak seen in the 1D model.

\section{$5 \quad$ Final remarks}

We have considered two models of the flow of hydrogen in a three-layer retina, extending the work of Farrow et al. [3] who considered a two-layer model. The 
inclusion of a third layer (vascular retina) is appropriate for the eyes of rats or humans, whereas the two-layer model is appropriate for guinea pigs [12, 13]. It is shown that the inclusion of the vascular flow layer can significantly alter the final velocity of the hydrogen peak value. Three separate well-mixed layers representing the choroid, avascular retina and vascular retina were considered in the 1D model. The diffusion $\alpha$ from the choroid to the avascular retina and then the vascular retina has a significant impact on the time required to reach the terminal velocity of the peak concentration. If $\alpha$ is small, the time taken until the change in the maximum peak position is longer and involves a sharper jump, while if $\alpha$ is larger this occurs earlier and more smoothly. The results indicate that the velocity of the peak in hydrogen concentration is not the same as the speed of the blood flow in the choroid, and so care is needed when interpreting the experiments. A formula was derived for the velocity of the peak concentration in the choroid, corresponding to the average mass flux in the whole three-layer system rather than the flow in the choroid alone. The results obtained from the $2 \mathrm{D}$ model of Section 4 verify the qualitative behaviour obtained from the simpler 1D model, demonstrating that the major features of such flows can be gleaned from the one dimensional, well-mixed layer model.

\section{References}

[1] V. A. Alder, D. Y. Yu, S. J. Cringle and E. N. Su. "Experimental approaches to diabetic retinopathy". Asia-Pac. J. Ophthalmol. 4:20-25, 1992. C120

[2] J. C. Arciero, P. Causin and F. Malgoroli. "Mathematical methods for modeling the microcirculation". AIMS Biophys. 4:362-399, 2017. doi:10.3934/biophy.2017.3.362 C120

[3] D. E. Farrow, G. C. Hocking, S. J. Cringle and D.-Y. Yu. "Modeling Hydrogen clearance from the retina". ANZIAM J. 59:281-292, 2018. doi:10.1017/S1446181117000426 C120, C133 
[4] A. B. Friedland. "A mathematical model of transmural transport of oxygen to the retina". Bull. Math. Biol. 40:823-837, 2018; doi:10.1007/BF02460609 C120

[5] D. Goldman. "Theoretical models of microvascular oxygen transport to tissue". Microcirculation 15:795-811, 2008. doi:10.1080/10739680801938289 C120

[6] A. C. Hindmarsh. "ODEPACK, A Systematized Collection of ODE Solvers". In Scientific Computing, R. S. Stepleman, et al., Eds., pp. 55-64. North-Holland, Amsterdam, 1983. C122

[7] S. S. Kety. "The theory and applications of the exchange of inert gas at the lungs and tissues". Pharmacol. Rev. 3:1-41, 1951. http://pharmrev. aspetjournals org/content/3/1/1 C120

[8] B. P. Leonard. "A stable and accurate convective modelling procedure based on quadratic upstream interpolation". Comput. Methods Appl. Mech. Eng. 19:59-98, 1979. doi:10.1016/0045-7825(79) 90034-3 C129

[9] S. L. Mitchell. Coupling transport and chemistry: numerics, analysis and applications. PhD thesis, University of Bath, UK, 2003.

https://researchportal.bath.ac.uk/en/studentTheses/ coupling-transport-and-chemistry-numerics-analysis-and-applic C127

[10] G. A. Winchell. Mathematical model of inert gas washout from the retina: evaluation of hydrogen washout as a means of determining retinal blood flow in the cat. Master's Thesis, Northwestern University, Evanston, USA, 1983. https://search.library.northwestern.edu/ permalink/f/5c25nc/01NWU_ALMA21563278530002441 C120

[11] D. Y. Yu, V. A. Alder and S. J. Cringle. "Measurement of blood flow in rat eyes by hydrogen clearance". Am. J. Physiol. (Heart Circ. Physiol.) 261:H960-H968, 1991. doi:10.1152/ajpheart.1991.261.3.H960 C120 
[12] D. Y. Yu, S. J. Cringle, V. A. Alder, E. N. Su, and P. K. Yu, "Intraretinal oxygen distribution and choroidal regulation in the avascular retina of guinea pigs". Am. J. Physiol. (Heart Circ. Physiol.) 270:H965-H973, 1996. doi:10.1152/ajpheart.1996.270.3.H965 C120, C133

[13] S. Cringle, D.-Y. Yu, V. Alder, E.-N. Su, and P. Yu. "Choroidal regulation of oxygen supply to the guinea pig retina". In A. G. Hudetz, and D. F. Bruley (Eds.), Oxygen Transport to Tissue XX, pp. 385-389. Springer, 1998. doi:10.1007/978-1-4615-4863-8 C120, C133

\section{Author addresses}

1. W. F. Mansoor, Mathematics and Statistics, Murdoch University, Murdoch, WA, 6150 Australia.

mailto:wafaa.mansoor2@murdoch.edu.au orcid:0000-0002-8992-0190

2. G. C. Hocking, Mathematics and Statistics, Murdoch University, Murdoch, WA, 6150 Australia.

mailto:g. hocking@murdoch.edu.au orcid:0000-0002-5812-6015

3. D. E. Farrow, Mathematics and Statistics, Murdoch University, Murdoch, WA, 6150 Australia.

mailto:d.farrow@murdoch.edu . au orcid:0000-0002-4716-7698 